\title{
Interaction Between Cardiac Myocytes And Fibroblasts: In Vivo And In Vitro
}

\author{
T.K. Borg, J.L. Hastings and C.A. Fix
}

Department of Cell and Developmental Biology and Anatomy, University of South Carolina, School of Medicine, Columbia, SC 29208

The cellular organization of the heart consists primarily of myocytes, fibroblasts, and endothelial cells. Myocytes make up the largest cellular volume; however, fibroblasts are the most numerous cell type (1). While endothelial cells are confined to the endocardial surface and lining of blood vessels, fibroblasts are interspersed within the layers of cardiac myocyte. Much is known about the organization of the cardiac myocytes and the vasculature; however, relatively little is known about the organization of the cardiac fibroblasts and their interaction with cardiac myocytes.

In addition to the cellular organization of the heart, the extracellular matrix (ECM) is composed of the acellular components surrounding the myocytes. These components include large molecular weight collagens, proteoglycans, glycoproteins, proteases, and small molecular weight matrikines and growth factors (2). After birth, these components are primarily secreted by the fibroblast. The organization of the ECM is arranged in a precise, 3-Dimensional network that is intimately associated with cardiac function (3). Recently, the organization of the fibroblasts was describe as an interconnected network of cells which was in contact with both the acellular ECM components and the cardiac myocytes $(4,5)$.

The goal of these studies was to further elaborate on the organization of this network of fibroblasts and its association with myocytes. In vitro studies were also undertaken to determine the function(s) of the fibroblast-myocyte interaction.

Hearts from adult (>30 days) rats were prepared for confocal microscopy as previously described (4). Fibroblasts were stained with the specific marker DDR2 $(3,4)$ and myocytes with phalloidin. Antibodies against connexin 43 and 45 were used to delineate the gap junctional compelexes. For in vitro studies, cardiac myocytes were isolated from 3-4 day old neonatal rat hearts as previously described and cultured on aligned collagen (6). For cell-cell interaction studies, antibodies were made in rabbits against the detergent soluble membrane fraction of neonatal fibroblasts. These antibodies were assayed to inhibit the interaction of myocytes and fibroblasts.

Cardiac fibroblasts are organized in a 3-D network within the ECM both in vivo and in vitro. The fibroblast exhibit a cell-cell interaction that is regulated in part by cadherin 11. Fibroblasts-ECM interactions are regulated by integrin and DDR2 receptors. The organization of the myocytes and fibroblasts was qualitatively similar both in vivo and in vitro. Quantitative differences in the amount of ECM, principally collagens, were evident from in vivo to in vitro. Mechanical stretching of the in vitro cultures showed increases in cardiac myosin and actin.

To determine if functional gap junctions occurred between myocytes and fibroblasts, fibroblasts were loaded with CMFDA, a low molecular weight fluorescinated compound and incubated with cardiac myocytes. Evidence of movement of the CMFDA from the fibroblasts to myocytes was evident (fig. 1). Movies of this contact site showed that these connections were discrete spots. Loading fibroblasts with larger dextrans did not show similar evidence. 
Cell-cell adhesion studies indicated that fibroblasts and myocytes formed contacts during culture conditions. Antibodies against the detergent soluble fibroblast membrane fraction inhibited this interaction (fig. 2). Further evidence of a myocyte-fibroblast interaction was seen by plating myocytes followed by fibroblasts under conditions that would result in a variety of phenotypes. In these studies, the fibroblasts always mimicked the pattern established by the myocytes. When the process was reversed, plating fibroblasts followed by myocytes, similar results were observed.

From these studies, we conclude that fibroblasts are organized in a 3-D network with contacts to the ECM, cardiac myocytes, and to each other. These contacts are regulated by different receptor families including integrins, cadherins, gap junctional proteins and DDR2. The contact between fibroblasts and myocytes has functional implications in patterning, ECM generation and turnover, and other physiological roles.

\section{Reference:}

1. H.W. Vliegen et al., Eur. Heart J. (1991): 12:488

2. T.K. Borg and J.B. Caulfield Fed. Proc. (1981) 40:2037.

3. E.C. Goldsmith et al., Develop. Dynamics (2004) 230:787.

4. P. Camelliti et al., Cardiovascular Res. (2004)

5. P. Camelliti et al. Circ. Res. (2004) 94:828.

6. $\quad$ D.G. Simpson et al., J Cell Physiol. (1994) 161:89.

7. This research was supported by NIH grants HL-068038 and HL-72160.

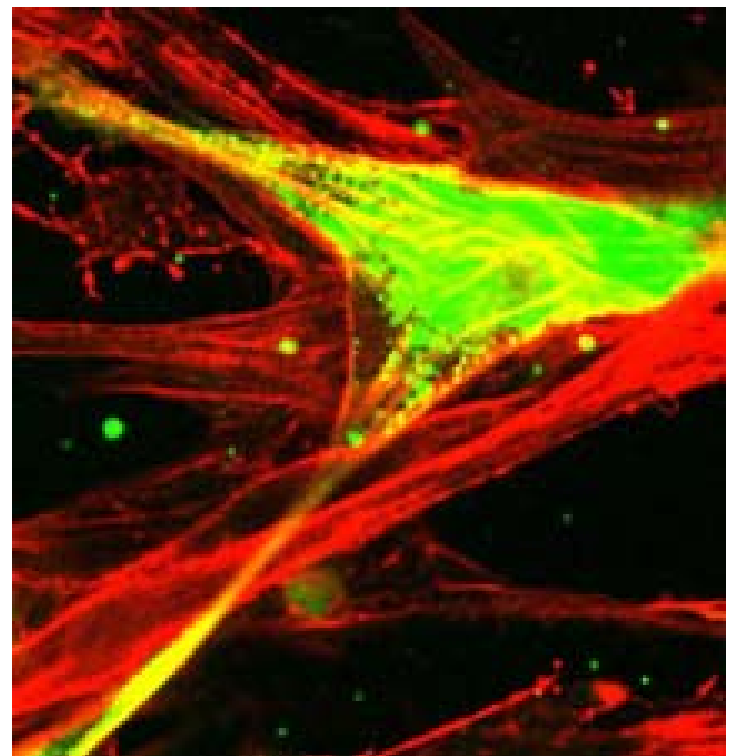

Figure 1

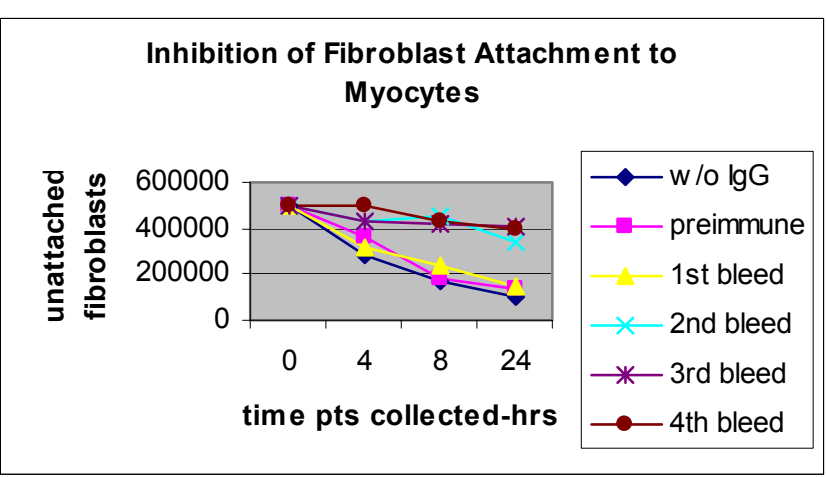

Figure 2 